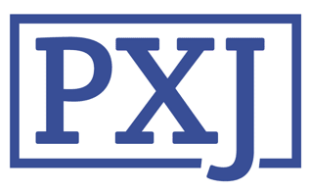

Patient Experience Journal

Volume 5 | Issue 1

Article 12

2018

\title{
Nursing transfer of accountability at the bedside: partnering with patients to pilot a new initiative in Ontario community hospitals
}

\author{
Kristina BA Miller \\ Western University \\ Aden Hamza \\ Western University \\ Kateryna Metersky \\ Western University \\ Dianne M. Gaffney \\ Huron Perth Healthcare Alliance
}

Follow this and additional works at: https://pxjournal.org/journal

Part of the Health and Medical Administration Commons, Health Policy Commons, Health Services Administration Commons, and the Health Services Research Commons

\section{Recommended Citation}

Miller KB, Hamza A, Metersky K, Gaffney DM. Nursing transfer of accountability at the bedside: partnering with patients to pilot a new initiative in Ontario community hospitals. Patient Experience Journal. 2018; 5(1):90-96. doi: 10.35680/2372-0247.1163.

This Research is brought to you for free and open access by Patient Experience Journal. It has been accepted for inclusion in Patient Experience Journal by an authorized editor of Patient Experience Journal. 


\section{Nursing transfer of accountability at the bedside: partnering with patients to pilot a new initiative in Ontario community hospitals}

\section{Cover Page Footnote}

This project was generously supported by the Canadian Foundation for Healthcare Improvement. The Canadian Foundation for Healthcare Improvement identifies proven innovations and accelerates their spread across Canada by supporting healthcare organizations to adapt, implement and measure improvements in patient care, population health and value-for-money. CFHI is a not-for-profit organization funded through an agreement with Health Canada. The views expressed herein do not necessarily represent the views of Health Canada. 


\title{
Nursing transfer of accountability at the bedside: partnering with patients to pilot a new initiative in Ontario community hospitals Kristina Miller, MSc, PhD (candidate),Western University, kmille46@uwo.ca Aden Hamza, RN, MN(c), Western University, abamza2@uwo.ca Kateryna Metersky, RN, MN, PhD (candidate),Western University, kaksench@uwo.ca Dianne Gaffney, MSc., RD, Huron Perth Healthcare Alliance,Dianne.gaffney@lhsc.on.ca
}

\begin{abstract}
The transfer of accountability (TOA) for a patient from one nurse to another at change of shift is an important opportunity to exchange essential patient care information, as well as to enhance the safety and quality of patient care. This study was undertaken to explore nurses', patients' and family members' perceptions associated with the implementation of bedside nurse to nurse TOA. Focus groups were conducted pre-implementation (two with nurses and two with patients and family members) and post-implementation (six with nurses and two with patients and family members). The focus groups were audio-recorded, transcribed and analysed using directed content analysis. Findings were divided into positive outcomes and challenges to bedside nurse to nurse TOA. Positive outcomes included increased patient safety, more informed patients more consistent use of whiteboards in the patient rooms, better engagement with family via the whiteboard and increased family involvement, confirmation of information between nurses, increased accountability between nurses, and personal introduction/icebreaker of the new nurse. The inclusion of the Patient Partners on the project team was a key success factor for the project. Challenges included a perception of lengthened time required for TOA and increased workload, lack of privacy and potential breaches of confidentiality, patient fear and lack of comprehension, lack of clarity in TOA processes, and inconsistent application of the procedures. Hospital administrators and nurse leaders can use these findings to anticipate and understand change associated with bedside TOA as seen by both nurses and patients/families.
\end{abstract}

\section{Keywords}

Transfer of accountability, nursing, qualitative methods, patient engagement, communication, patient experience

\section{Introduction}

Fundamental to safe quality patient care is effective communication occurring between healthcare providers. ${ }^{1-3}$ In fact, $70 \%$ of all sentinel events occur as a result of communication breakdown. ${ }^{4}$ Many hospitals in Canada do not have consistent standards or policies in place for a change of shift handoff, resulting in significant variances in transfer of accountability (TOA) practices between and within health organizations. These varied practices can include but are not limited to, taped reports, verbal exchanges between nurses away from the bedside, reading of medical records, and end-of-shift reports generated from nursing documentation in the electronic health record. ${ }^{5-11}$ Such exchanges occur in a conference room or at the nursing station, providing no opportunity for patient or family to be involved and contribute to the discussion. ${ }^{12}$ A 2014 Cochrane systematic review which sought out to find which nursing handover style works best, could not make a definitive conclusion as there were no randomized controlled studies on the topic. ${ }^{11}$ However, the review did mention four guiding principles that can be applied when designing or implementing a nursing handover process, two of which were patient involvement and face-to-face communication. ${ }^{11}$

\section{Background}

Patient bedside TOA is poorly defined in healthcare settings. ${ }^{13}$ Bedside TOA from one nurse to another at change of shift is an important opportunity to communicate vital patient care information such as patient diagnosis, hemodynamic stability, procedures performed, plan of care, discharge plans and information to bring forward to interprofessional rounds, while at the same time engaging the patient in the process. ${ }^{14}$ Bedside TOA ensures a smooth, seamless transition for all involved as well as enhances the safety and quality of patient care.

The benefits of bedside TOA as include increased patient safety (reduction in adverse events such as medication errors, pressure ulcers and falls) ${ }^{12}$, enhanced patient satisfaction ${ }^{19}$, and trust in nurses' professionalism and competence ${ }^{20}$ as patients now 
have a clearer understanding of the nurses' role in their care. ${ }^{21}$ Patients have access and input into their plan of care through engagement in this exchange, ${ }^{2}$ and reduced anxiety by a personalized and timely introduction to the oncoming nurse and the opportunity to ask questions. ${ }^{12}$ Bedside TOA increases staff satisfaction. It allows presents nurses with that same ability to ask questions of the patient and outgoing nurse in a timely fashion, increasing accountability and interpersonal relationships. ${ }^{17,19,21}$ Also, at the bedside, the nurse can conduct a visual inspection of the patient and surrounding environment. ${ }^{21}$ It allows for a more effective prioritization of patient care in the oncoming shift as well as to confer with the outgoing nurse regarding any discrepancies between what is observed and visualized. ${ }^{21}$ Staff physicians even report increased satisfaction with nurse bedside TOA as they find nurses better informed and able to respond to questions regarding the patient shortly after shift change. ${ }^{19}$

Despite these benefits, bedside TOA is a difficult change to implement. Nurses have reported some challenges, including concerns regarding violating patient confidentiality (with patients in semi-private or ward accommodations). ${ }^{21,} 22$ However, nurses, for the most part, can discern what was appropriate to share at the patients' bedside. ${ }^{16}$ Other challenges include a lack of confidence and comfort in talking in front of patients leading to an increase in anxiety and having a perception that the process is more time-consuming than other methods of TOA, especially if patients start asking questions. $3,14,21,23$ Interestingly, a number of authors found that bedside TOA implementation actually decreased overtime hours by 100 hours in the first two pay periods as it took 2-5 minutes on average per patient. 9, 14, 19, 24 This, in turn, equated to lower healthcare spending on unnecessary overtime.

In relation to patient reported challenges with bedside TOA, patients varied in their desire to participate in this form of shift handover, ${ }^{15}$ despite bedside TOA's alignment with patient-centered care. ${ }^{25-28}$ On family participation during bedside TOA, none of the previous studies have evaluated the impact of family inclusion. This study differs from previous studies on this topic by including patient and family partners, along with staff and leaders on the project team, in the design, implementation and evaluation of a pilot project of bedside TOA. Interestingly, a 2016 systematic review of articles published between 2008 and 2014 on bedside TOA found that only 6\% of these articles looked at or studied patient outcomes to evaluate bedside TOA. ${ }^{29}$ Thus, the aim of this study was to improve the quality and safety of patient care and the patient experience through engagement of patients and their family members in the exchange of information at change of shift at the bedside.

\section{Methods}

This pilot study used a mixed method evaluative research design, comprising of both quantitative and qualitative strategies, to assess the implementation of bedside TOA between nursing staff that included patient and family engagement. The project team included registered nurses (RNs), registered practical nurses (RPNs) and leaders from the two pilot units, as well as a patient partner, a family partner, the Corporate Lead Professional Practice and the Director of Decision Support. The executive sponsor for the project was the Vice President of Partnerships and Patient Experience. This team designed the processes for bedside TOA based on the SBAR format (Situation, Background, Assessment, Recommendations), education sessions for nursing staff on the pilot units in which the patient partners actively participated, as well as the methodology and evaluation strategy for the study.

\section{Settings and participants}

The study was conducted by the Huron Perth Healthcare Alliance (HPHA), which is an alliance of four community hospitals in Huron and Perth Counties in Southwestern Ontario, serving a population of 174,000 . The study was piloted on the Inpatient Medicine/Complex Continuing Care Unit at St. Marys Memorial Hospital (SMMH) and the Inpatient Surgery unit at Stratford General Hospital (SGH). SMMH is 20-bed acute and complex continuing care hospital while SGH is an 113-bed acute care hospital. Study participants included RNs and RPNs currently working on each unit, as well as patients and their family members. Patients who were less than 18 years of age, or who were unable to communicate in English (verbally and in writing) were ineligible for participation in the study. The Research Ethics Board of Western University, London, Ontario, Canada provided ethics approval. All patients, family members and staff who participated in the study provided informed consent. A letter of information was provided along with a verbal explanation of the study, following which written consent was obtained.

\section{Data collection and analysis}

Qualitative data was collected twice; the preimplementation (T1) focus groups took place in March 2015 and post-implementation (T3) in September 2015. The use of focus groups to collect qualitative data allows for a deeper understanding of the impact and issues of bedside TOA for both staff and patients and family members. Quantitative data was collected preimplementation (T1), three months post-implementation (T2) and six months post-implementation (T3). This paper will focus on the qualitative findings derived from focus groups of staff, patients and family members before and 
after bedside TOA was implemented, with some supplemental quantitative findings presented for support.

All focus groups took place at their respective hospitals. Staff and leaders invited patients and their family members on the unit to participate in the study on the day of and up to two days before the focus groups. Nursing staff were notified of the date of focus groups and invited to participate. The manager on the unit brought in an additional nurse to cover staff patient assignments during the focus groups. Questions for patients and families centred around accessing information about patient care, the type of information given, preferred types of communication, level of engagement and patient satisfaction with nursing TOA. Similarly, questions for nurses included their experience in TOA, where they get their best information, how to improve patient and family communication and engagement.

A total of 44 participants took part in the 13 focus groups (33 staff; eight patients; three family members), with the number of participants in each group ranging from two to seven people. At T1, two staff focus groups (SGH - six staff; SMMH - seven staff) and two patients/family focus groups (SGH - two patients, one family member; SMMH three patients) were conducted at each site. At T3, two staff focus groups were conducted at SGH (nine staff) and three focus groups at SMMH (11 staff). One

patient/family focus group was conducted at each hospital site (SGH- two patients, one family member; SMMH - one patient, one family member) during this period. A sample of convenience was used as the number of available participants was dependent on the staffing needs of the unit and the appropriateness of the patient/family population.

All focus groups were conducted by one researcher (AH) and followed a semi-structured interview guide. The focus groups were tape-recorded and transcribed verbatim. The transcriptions for T1 data were coded independently using directed content analysis ${ }^{30}$ by two researchers (AH and $\mathrm{KM}$ ), followed by consensus-coding where codes were compared, key themes were identified, and findings were developed jointly. The same process was followed for analysis of the T3 data.

\section{Results}

\section{Positive outcomes with the change in nurse to nurse bedside TOA}

There were potential positives identified during the T1 focus groups with staff, patients and family members that were confirmed in the T3 focus group, with additional positives also being identified. Potential positives included increased patient safety, more informed patients, more consistent use of whiteboards in the patient rooms, better engagement with family via the whiteboard, and better family involvement in TOA where they could confirm information as well as provide additional information not obtained through other assessments. Newly identified positives included confirmation of information between nurses, increased accountability between nurses and personal introduction/ice breaker with a new nurse.

Increased patient safety

The emphasis on increased patient safety centres on the joint "laying of eyes" on the patient by the outgoing and oncoming nurse, ensuring a greater knowledge transfer of the patient's condition at that point in time. This benefit was first identified in the'T1 focus groups:

"But I think it would be beneficial for the nurse and the other nurse, they both setting eyes on that patient so that you can't call them, 2 hours, 20 minutes later and say ob my gosh, so-and-so, you know, what happened?" [Nurse 3, Site 2]

Increased patient safety via joint "laying of the eyes" by the oncoming and off-going nurse was highlighted in almost every T3 focus group with the nursing staff:

"...it's nice seeing the patient how [other nurse] leaves so we both eyeball the patient at the same time, this is how you left him, and I know like if I went in half an hour later and we didn't do that, is that what the patient looked like when [the other nurse] left."

[Nurse 1, Site 1]

More informed patient

Participants recognized that bedside TOA carried potential benefits for nurses, patients and families. Patients found access to information through bedside TOA empowering and reassuring and valued the opportunity to contribute to their plan of care. Nurses also recognized the importance of patients being informed about their health and the staff caring for them.

"Information is power. And it makes you feel better... I understand what's going on and then I don't, if something should go wrong, then I know whether it's something that needs attention or it's something that I can just wait or whatever. Don't go all crazy." [Patient 1, Site 1]

Nurses recognized post-intervention that patients were more informed:

"I think it's good for both the patients [and the nurse] so they know kind of what's going on..." [Nurse 11, Site 1]

More consistent use of whiteboard/better engagement with family via whiteboard

While whiteboards were already present in patient's rooms, they were not used consistently by staff and the 
information was not always up-to-date or correct. By incorporating the use of the whiteboard in the intervention, more consistent use of the whiteboards occurred, resulting in better family engagement and communication.

"We put questions on there [the whiteboards] sometimes, or the family does ... so that's another way of communicating our family's wishes for bathing and things like that, or clothes...so we kind of incorporate it in the transfer of accountability and write our name on and stuff, so it has become part of our transfer of accountability."

[Nurse 10, Site 2]

"I think they're [the whiteboards] used more now with the [bedside] TOA...having it there for the patient questions, and whatnot is great... sometimes we [were] not catching the family" [Nurse 16, Site 2]

Better family involvement where they can provide, confirm and fill in information gaps

Having patients' family members involved in bedside TOA allowed the family to verify the accuracy of information provided by the patient and fill in gaps as needed for hospital staff.

"I really liked it today at report because the patient's husband was there and so there was the five of us at the bedside, and then the patient said this is an excellent communication tool...So, I just thought it was an excellent opportunity for everybody involved to summarise the day and see what is next to go forward." [Nurse 8, Site 2]

"As being the spokesperson, you know, for a parent, it's very important to be aware of changes in health, changes in procedure, comments staff have about patients' quality of health, wellbeing, as well - that's important to us as a family member. As I said we're not here 24 hours a day, we're only here for a short window... but it's very important to us a family member to know what is happening when we're not here." [Family member 1, Site 2]

Confirmation of information between nurses

Having the TOA occur in front of the patient allowed them to confirm information as it was being communicated between the oncoming nurse and the outgoing nurse. It also provided the patient with an opportunity to communicate any information they saw as important to the oncoming nurse as they began their shift.

"I think it's good [engaging patients in their care] because then they know what's going on and they can have a say about how things are done - what they like and don't like, and ask questions." [Nurse 12, Site 2]
"I think for the most part I found that they just listen to what we say. And every once in a while they interject and "Oh, and don't forget."...Even when you say at the end, I said: "Is there anything else you want to add?" They say "No, that's good."' [Nurse 15, Site 1]

Introduction/Icebreaker with new nurse A benefit of the bedside TOA process was the personal introduction of the oncoming nurse, providing an icebreaker for the patient to facilitate communication with their new nurse.

"It is better that your first meeting is a hi, hello, I'll be right back then we're going to put you on the toilet and I've never talked to you before...you might be a little uncomfortable with them seeing your bits and pieces, and you haven't even talked to them before." [Nurse 17, Site 2]

"[I like knowing who my nurse is]. She becomes a person, or he becomes a person that way and it's easier to connect with somebody." [Patient 4, Site 2]

Overall, there were many positives associated with the change to bedside TOA, including those predicted by nursing staff before the intervention and others that were identified after the intervention was implemented.

\section{Challenges to Bedside TOA}

Some challenges in the implementation of bedside TOA were identified at both T1 and T3, as well as some unanticipated challenges. Challenges included a perception of lengthened time required for TOA and increased workload, lack of privacy and potential breaches of confidentiality, patient fear and lack of comprehension, while the new challenges of lack of clarity in TOA processes and inconsistent application of the procedures were identified solely in T3 focus groups.

Lengthened TOA time and increased workload One of the most common challenges identified by both staff and patients at T1 was the anticipated increased length of time that bedside TOA would require as compared to their current methods, and how the process would impact the staff's workload. Staff expressed concern that bedside TOA may encourage patients to seek direct care (e.g. assistance with toileting) during the shift report adding time to their shift report and decreasing efficiency.

"... first thing in the morning I say is a busy time. You have people who have to have bed pans for first thing in the morning after they've been asleep all night you know." [Patient 1, Site 1]

Post-intervention, nurses still saw the struggle and the benefit: 
"They want up, they want their wash, they want this, they want that, they want - I mean certain things you can do in that TO A, but you can't give a whole bed bath and that's what they're expecting."

[Nurse 16, Site 2]

"...it is hard to take the time and go to every patient's room and do a thorough, an effective TO A...but I think it is a good idea for sure." [Nurse 12, Site 1]

In actuality, quantitative assessments of the time required for change of shift report revealed that bedside TOA reduced the time required by approximately 4 minutes on average (baseline $=28.5$ minutes; post-implementation of bedside TOA $=24.8$ minutes), and the average time per patient was 2 to 3 minutes. A variable to be noted is that pre- and post-implementation assessments involve different patients since the average length of stay on acute care units is 3 to 5 days. The same staff members were included in the pre- and post-implementation assessments.

Lack of Privacy and Potential Breaches of Confidentiality A common challenge mentioned in staff focus groups both at T1 and T3 was the lack of privacy at the patient's bedside, resulting in potential breaches of confidentiality. Staff expressed concern about discussing patient information during bedside TOA as it would expose the health information of the individual to others in the room. Following the implementation of bedside TOA, staff found that patients easily overheard the information shared, which concerned staff as they wanted to engage in communication with patients, but felt that this weakened their ability to maintain privacy and confidentiality of patients.

"It's not right because we're going to be disclosing to other people and that's one of our first things as part of the [hospital] and as our College of Nurses and our practice standards, standards of practice, is to provide privacy and confidentiality.' 'Nurse 6, Site 2]

"I have big issues with the confidentiality. You know, they don'tthose curtains don't stop any sound." [Nurse 7, Site 1]

To help mitigate these risks, obtaining consent from the patient was included in the bedside TOA procedures developed for the project. In practice, staff adapted their report to ensure sensitive information was discussed outside the patient's room before bedside TOA, thus decreasing the risk of breaching confidentiality with pertinent patient information.

"We chose to talk outside the room, depending on what the information was, whether it was a new diagnosis or something like that. So not often but once in a while, we choose to finish or continue outside the room." [Nurse 11, Site 2]
Patient Fear and Lack of Comprehension

In the T1 focus groups, staff identified that bedside TOA might allow for patients and families to misinterpret the information being communicated (i.e. laboratory values and other assessments), which may incite fear and undue concern. Also, they were concerned that the amount of information communicated might overwhelm some patients and families.

"Because they're not going to understand numbers and things like that right? And you can try to explain things to them... I don't think. it's going to get you anywhere." [Nurse 4, Site 1]

After initiating bedside TOA, staff identified variability in patients' ability and willingness to actively engage and benefit from bedside TOA. Staff identified factors such as cognition; hours slept and timing of shift report that influenced the appropriateness of engaging patients in bedside TOA.

"I think it's hard when people are upset at us for not waking up patients at $7 o^{\prime}$ clock in the morning when a lot of them are confused or demented...I find that challenging." [Nurse 17, Site 2]

"I think the whole [bedside] TOA really needs to correlate closely with the patient population." [Nurse 18, Site 2]

$\underline{\text { Lack of clarity in TOA processes and inconsistent }}$ application

The staff identified the unanticipated challenge of variability in expectations for the content and purpose of bedside TOA in the T3 focus groups. Also, staff, patients and family members identified a lack of consistency in the application of bedside TOA, leaving some patients and family disengaged from the process.

"There's some stuff that does need to be said at the bedside either if you look at the SBAR, it's way too much information to actually sit there and go, it's not necessary. It should be just a brief little overview, it doesn't have to be that whole SBAR thing." [Nurse 10, Site 1]

In summary, the $\mathrm{T} 1$ focus groups participants reflected on the previous change-of-shift report as well as perceived challenges to implementing the proposed bedside TOA. T3 focus groups identified that some of these challenges were realized in implementing effective bedside TOA, although, on the concern about increased time requirements for bedside TOA, this appears to be a misperception as the quantitative assessments actually revealed a shorter time requirement. In response to these challenges, participants did identify some strategies to mitigate them, such as ensuring patient choice, determining the appropriateness of the patient to partake 
bedside TOA and discussing sensitive information outside patient rooms.

\section{Discussion}

This study provides an analysis of nurses', patients' and family members' experiences and perceptions of bedside nursing TOA and expands upon current evidence in this area by including both nurses and patients/family members within the same study, as well as patient/family partners on the project team. Some of the positive outcomes of bedside TOA implementation reported in this study confirm previous reports in the literature, such as an increase in patient safety, a personalized and timely introduction to the oncoming nurse, as well as the ability for patients and family members to ask questions. ${ }^{12,23}$ These benefits were similar to the findings of another study in which staff satisfaction was positively impacted by bedside TOA, as it provided nurses with the opportunity to visualize the patient and ask questions of the outgoing nurse and patient in a timely fashion. ${ }^{19,} 23$ Regarding findings not previously reported in the literature, this study highlights the added value of involvement of family in bedside TOA, as well as the impact of bedside TOA on increased use of patient whiteboards for enhanced written communication between staff, patients and family members. In terms of perceived challenges, lengthened TOA time, increased workload and lack of privacy leading to potential breaches of confidentiality were previously reported in the literature. $3,9,14,16,19,23,24$ The nurses also noted that they used their clinical judgement with respect to sharing sensitive information at the bedside, particularly if the patient was in a semi-private or ward room. Such information would be discussed with the patient when other patient's visitors weren't present, and when the nurse had more time to spend with the patient. This study revealed an additional concern about patient fear due to an anticipated lack of comprehension of information shared during the report, as well as staff concerns postimplementation about the lack of clarity in bedside TOA processes and inconsistent application. The latter concern was due in part to confusion amongst staff regarding what information should be included in their documentation in the electronic health record, and what information should be included in the bedside TOA report, with a desire to avoid duplication. This highlights the importance of ongoing education, support and mentoring of staff following the initial implementation until the staff are comfortable with the process, and it is embedded in their practice. Jeffs and colleagues ${ }^{23}$ noted the importance of providing inservices for staff, visible support from corporate leaders as well as sharing feedback from staff and patients with staff. The post-implementation findings support their recommendations, and in addition to the two-hour inservices (which included patient partners) that were provided to all nursing staff on the pilot units preimplementation, unit and corporate leaders supported staff at change of shift for several months post-implementation, and feedback from staff and patients/family members was shared on huddle boards (i.e. unit communication boards). The inclusion and full engagement of patients and family along with staff and leaders on the project team throughout all stages was a significant factor in the success of this study, as their opinions and concerns were incorporated into the design of procedures, education and evaluation strategies.

\section{Study Limitations}

The transferability of the study findings is limited in two ways. The first is the low number of patient and family members recruited for the focus groups, particularly the T3 focus groups. These participants were difficult to recruit as they were same day efforts due to the changing nature of patients on the units. The second is that staff members indicated they were experiencing change fatigue due to the number of changes occurring within their organization, which may have contributed to a more negative attitude about the change to bedside TOA.

\section{Conclusion}

This study highlights the benefits and challenges to implementing bedside TOA from the perspectives of both nurses and patients/family members. The study findings indicate that concerns such as increased time to conduct bedside TOA and patients not wanting to be involved in TOA (for example) were unfounded. As well, the level of engagement varies between patients, and the inclusion of patient consent is an important part of the process. Overall, many of the staff and patient comments cited the benefits of bedside TOA. Further work on elucidating the content and procedures included in bedside TOA will be of benefit in addressing this concern raised by nurses. Future projects should include patients, family members, front-line staff and leaders as members of the project team during all phases, to ensure the perspectives of all stakeholders are included in the design, implementation and evaluation of the project.

\section{References}

1. Forsyth S, Poole N, Alvarado K, et al. Transfer of Accountability: Transforming Shift Handover to Enhance Patient Safety. Healthc Q. 2006;9(Sp):75-79.

2. Chaboyer W, McMurray A, Johnson J, Hardy L, Wallis M, Chu FY. Bedside handover; Quality improvement strategy to transform care at the bedside. J Nurs Care Qual. 2009;24(2):136-142.

3. Evans D, Grunawalt J, McClish D, Wood W, Friese CR. Bedside shift-to-shift nursing report: implementation and outcomes. Medsurg Nurs. 2012;21(5):281-292. 
4. Joint Commission on Accreditation of Health Organizations. Sentinel event statistics 2003; http://www.jointcommission.org/SentinelEvents/Sta tistics.

5. Dowding D. Examining the effects that manipulating information given in the change of shift report has on nurses' care planning ability. J Adv Nurs. 2001;33(6):836-846.

6. Greaves C. Patients' perceptions of bedside handover. Nurs Stand. 1999;14(12):32-35.

7. Kerr MP. A qualitative study of shift handover practice and function from a socio-technical perspective. J Adv Nurs. 2002;37(2):125-134.

8. Pothier D, Monteiro P, Mooktiar M, Shaw A. Pilot study to show the loss of important data in nursing handover. BrJ Nurs. 2005;14(20):1090-1093.

9. Timonen L, Sihvonen M. Patient participation in bedside reporting on surgical wards. J Clin Nurs. 2000;9(4):542-548.

10. Williams AJ. Managing change in the nursing handover. Nurs Stand. 1998;12(18):39-42.

11. Smeulers, M., Lucas, C., \& Vermeulen, H. (2014). Effectiveness of different nursing handover styles for ensuring continuity of information in hospitalised patients. Cochrane Database of Systematic Reviews, 6, Art. No.: CD009979. doi: 10.1002/14651858.CD009979.pub2.

12. Maxson PM, Derby KM, Wrobleski DM, Foss DM. Bedside nurse-to-nurse handoff promotes patient safety. Medsurg Nurs. 2012;21(3):140-145.

13. Cohen MD, Hilligoss PB. The published literature on handoffs in hospitals: Deficiencies identified in an extensive review. Qual Saf Health Care. 2010;19(6):493497.

14. Caruso EM. The evolution of nurse-to-nurse bedside report on a medical-surgical cardiology unit. Medsurg Nurs. 2007;16(1):17-22.

15. Jeffs L, Beswick S, Acott A, et al. Patients' Views on Bedside Nursing Handover Creating a Space to Connect. J Nurs Care Qual. 2014;29(2):149-154.

16. Jeffs L, Acott A, Simpson E, et al. The value of bedside shift reporting enhancing nurse surveillance, accountability, and patient safety. J Nurs Care Qual. 2013;28(3):226-232.

17. Kerr D, Lu S, McKinlay L. Bedside handover enhances completion of nursing care and documentation. J Nurs Care Qual. 2013;28(3):217-225.

18. Sand-Jecklin K, Sherman J. Incorporating bedside report into nursing handoff: Evaluation of change in practice. J Nurs Care Qual. 2013;28(2):186-194.

19. Anderson CD, Mangino RR. Nurse shift report: Who says you can't talk in front of the patient? Nurs $A d m$ Q. 2006;30(2):112-122.

20. Lupieri, G., Creatti, C., \& Palese, A. (2015). Cardiothoracic surgical patients' experience on bedside nursing handovers: Findings from a qualitative study. Intensive and Critical Care Nursing, 35, 28-37.
21. Grimshaw, J., Hatch, D., Willard, M., \& Abraham, S. (2016). A qualitative study of the change-of-shift report at the patients' bedside. The Health Care Manager, 35(4), 294-304.

22. Anderson, J., Malone, L., Shanahan, K., \& Manning, J. (2015). Nursing bedside clinical handover - an integrated review of issues and tools. Journal of Clinical Nursing, 24, 5-6, 662-671.

23. Jeffs L, Cardoso R, Beswick S, et al. Enablers and barriers to implementing bedside reporting: insights from nurses. Nurs Leadersh. 2013;26(3):39-52.

24. Athwal P, Fields W, Wagnell E. Standardization of change-of-shift report. J Nurs Care Qual. 2009;24(2):143-147.

25. Aboumatar HJ, Cooper LA. Contextualizing patientcentered care to fulfill its promise of better health outcomes: Beyond who, what, and why. Ann Intern Med. 2013;158(8):628-629.

26. Bitzas V, Calestagne PP, McVey L, Lapointe B. Encouraging patients and families to influence change on a palliative care unit: Value of patient satisfaction surveys. J Nurs Care Qual. 2011;26(4):350-357.

27. Luxford K, Safran DG, Delbanco T. Promoting patient-centered care: a qualitative study of facilitators and barriers in healthcare organizations with a reputation for improving the patient experience. International journal for quality in health care: journal of the International Society for Quality in Health Care / ISQua. 2011;23(5):510-515.

28. Thomas L, Donohue-Porter P. Blending evidence and innovation: Improving intershift handoffs in a multihospital setting. J Nurs Care Qual. 2012;27(2):116-124.

29. Mardis, T., Mardis, M., Davis, J., Justice, E., Holdinsky, S., Donnelly, J., Ragozine-Bush, H., \& Riesenberg, L. (2016). Bedside shift-to-shift handoffs: A systematic review of the literature. Journal of Nursing Care Quality, 31(1), 54-60.

30. Hsieh H-F, Shannon SE. Three Approaches to Qualitative Content Analysis. Qualitative Health Research. 2005;15(9):1277-1288. 